\title{
Management and maintenance of TTCN Abstract Test Suites
}

\author{
Pierre-Yves Danet \\ France Télécom - CNET \\ Technopole Anticipa - 2 av. Pierre Marzin - 22307 Lannion Cedex, France \\ Tel: +339605 11 55 Fax: +3396053890 \\ E-mail: Pierre-Yves.Danet@lannion.cnet.fr \\ Etienne Desécures \\ Sema Group \\ 16 rue Barbès - 92126 Montrouge Cedex, France \\ Tel: +33140924092 Fax: +331473575 56 \\ E-mail: Etienne.Desecures@sema-taa.fr
}

\begin{abstract}
Production and maintenance of large Abstract Test Suites (ATSs) has become a complex activity involving several actors. This paper presents the main features of a method supporting this activity and describes a tool supporting this method. This tool, based on the Concerto platform, is being progressively used at CNET Lannion for the ISDN ATSs management.
\end{abstract}

\section{INTRODUCTION}

Today, ATSs are large and complex objects. They are difficult to set up. Their life cycle is long and a lot of modifications must be performed due to several external reasons:

- Standardization bodies never provide ATS actually validated. Corrections must be applied when ATS are really implemented and used on test tools.

- Standards themselves evolve. ATSs must be kept consistent with these evolutions.

All modifications must be controlled: people, particularly test equipment manufacturers, need to know precisely the modifications done on ATSs. Identified versions of ATSs must be periodically distributed to users. Trace of the evolutions must be kept. One should know when and why a modification has been done, which client has received what, etc. It is more or less a question of quality management.

Moreover, some ATSs use tables already existing in other ATS. In order to keep the different ATSs consistent and to avoid making the same modification several times, ATS fragments must be physically shared among ATSs.

The group in charge of the maintenance of ATSs used for checking the conformity with the ISDN protocol at CNET (France Telecom Research Centre) experienced this complex activity. 
It provides ISDN ATSs to test laboratories, equipment suppliers and standardization bodies (OSTC, ETSI, ...). The need for appropriate methods and tools has been clearly identified.

In this context, CNET developed a method for its ATSs management and maintenance and asked Sema Group to build a tool to support this method. This tool is based on the Concerto platform and particularly on its TTCN, Organizer and Configurator environments.

\section{TEST SUITES MANAGEMENT AND MAINTENANCE METHOD}

Once adopted by ad hoc standardization bodies (French or European), ATSs enter a maintenance process. ATSs are maintained by a managing site which distributes them to user sites for exploitation, e.g. test laboratories.

The maintenance process is essentially based on document exchanges between the managing site and the user sites and is organised in three main steps:

\section{Observation step}

An observation form, giving all necessary details, is issued whenever a user site detects a problem within an ATS. This problem may involve several ATS tables. The observation form is either transmitted by fax or through an electronic network to the managing site. An observation form is identified by the user site.

Every observation is acknowledged by the managing site. Usually processing an observation consists in performing some modifications to the related tables.

\section{Modification step}

Periodically, the managing site examines all the observation forms. Once modifications are achieved, the managing site informs the user sites by issuing a modification form.

The modification form indicates the corrected table(s), the ATSs, the nature of the correction made and refers to the related observation form. A modification form is also identified by the managing site.

Every change made to a table is described by a trace statement, that identifies clearly the modification and appears in the history list of the table. This history list is automatically inserted in the Detailed Comments field of the TTCN table.

\section{Delivery step}

When the managing site estimates that a sufficient number of changes have been made to an ATS or that a blocking point has been reached, it decides to perform a delivery to the user sites.

Two kinds of deliveries can be performed:

- A modification delivery is composed of the list of modification forms made on the ATS tables, and of the GR (graphical format) of these tables.

- A full ATS delivery is composed of the complete ATS GR, and of the set of modification forms made on the ATS tables since the last complete delivery.

In both cases, the ATS MP (machine processable format) is delivered on magnetic support. 


\section{METHOD SUPPORT: THE NUMERIX SOLUTION}

The software solution developed by Sema Group is called Numerix for it was first developed for the Numeris (France Telecom ISDN network) test suites.

The managing site is equipped with workstations running the Numerix tool, while user sites may (it is not mandatory) be equipped with a workstation running Concerto/TTCN so as stable versions of ATSs can be consulted. Being able to guarantee a frozen status of a suite across sites is a key feature of the solution, since it provides reliable information.

The Numerix solution is built upon the Concerto factory and makes use of:

- the Concerto/TTCN environment,

- the Organizer environment,

- the Configurator environment,

- the Doc environment.

Specific commands to assist the different maintenance steps are provided to the user.

The TTCN environment is composed of a TTCN editor, a PICS and PIXIT editor, a document generator, a static semantics checker, a quality checker and a specialized archive system allowing the management of large ATSs. ATSs can share common tables. Thus, when a table is modified, all ATSs providing that table are automatically updated.

The Organizer environment allows the management of the activities within a Concerto site. It allows creation, updating and management of tasks in conformity with rules defining a given method and submission of these tasks to the agents in charge of their execution.

The Configurator environment provides version and configuration management for the objects produced within the factory and in particular for ATSs and ATSs fragments.

The Doc environment is used to produce and manage technical documents.

The various components of the Numerix solution are used in the following way:

- the TTCN editor and associated semantic functions to produce and check the ATSs,

- the TTCN archive system to store the ATSs and manage the sharing of fragments between

ATSs,

- the Organizer to implement the observation and modification forms,

- the Configurator to supply version management on the ATSs.

Specific and simple commands allow to go from one step of the method to another: create an observation form, deliver modifications, generate a modification form, etc.

Observation and modification forms are implemented as tasks managed by the Organizer environment. A task represents an activity performed by one or several users and contains references to the data used and produced by the activity.

A task follows a precise life cycle: it is first created according to a task model, then submitted to a user, who starts, then completes the task.

It is possible to query the registered information through Organizer commands. For example it is possible to know all observations related to a given TTCN table. 


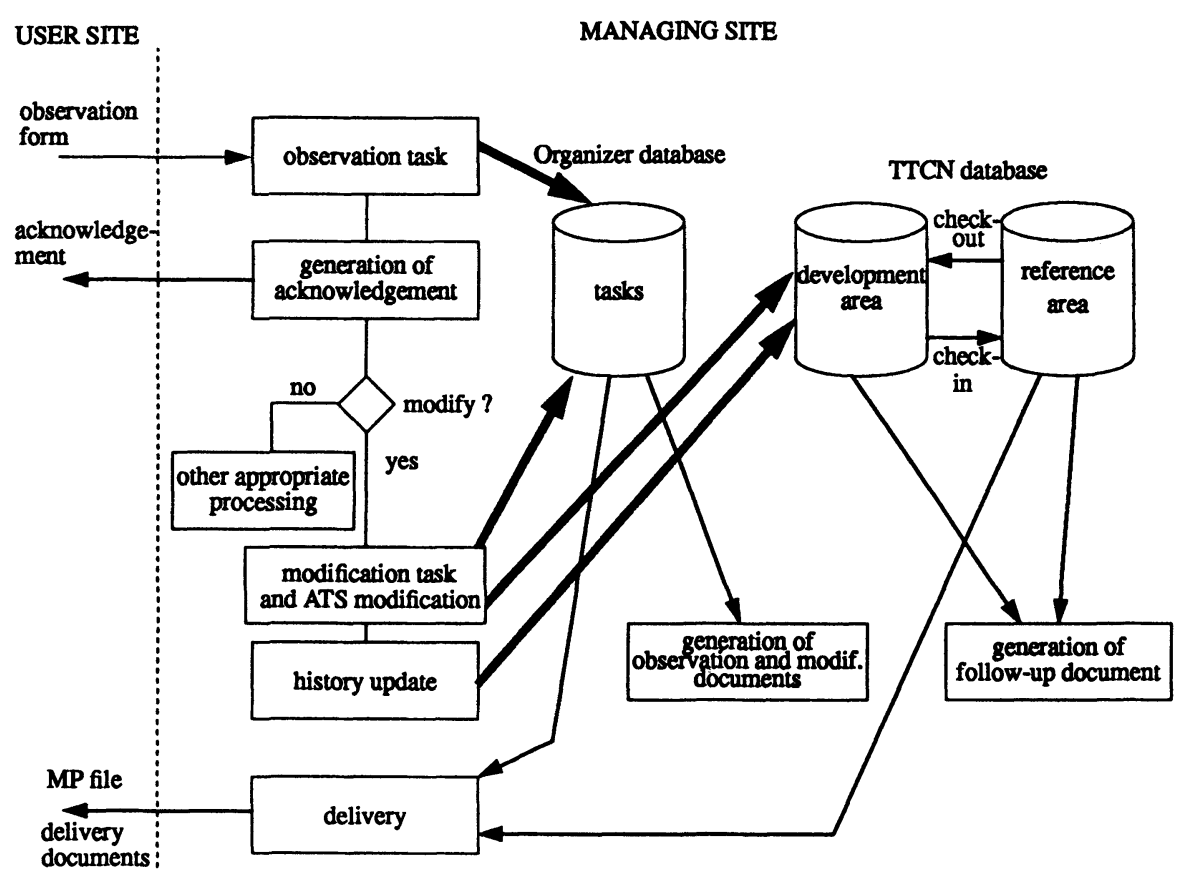

Figure 1 The Numerix solution

\subsection{Observation tasks}

The information contained in a user site observation form is reported manually within Numerix by the means of an observation task.

The TTCN tables mentioned by the observation form are referenced in the task by hypertext links set by the user. Their contents are thus directly accessible from the task.

The managing site specifies in this task how the observation will be processed, for example:

- do the modification,

- first do a complementary study,

- forward to concerned bodies,

- no processing.

Several observations issued from different user sites may concern the same tables on the same subject. Such observations are called associated and lead to a unique processing. These associations are implemented by hypertext links in the associated observations field.

The Numerix tool can be used to produce a document from an observation task. This document, including the answer elements, can be sent to the issuing site as an acknowledgement. 


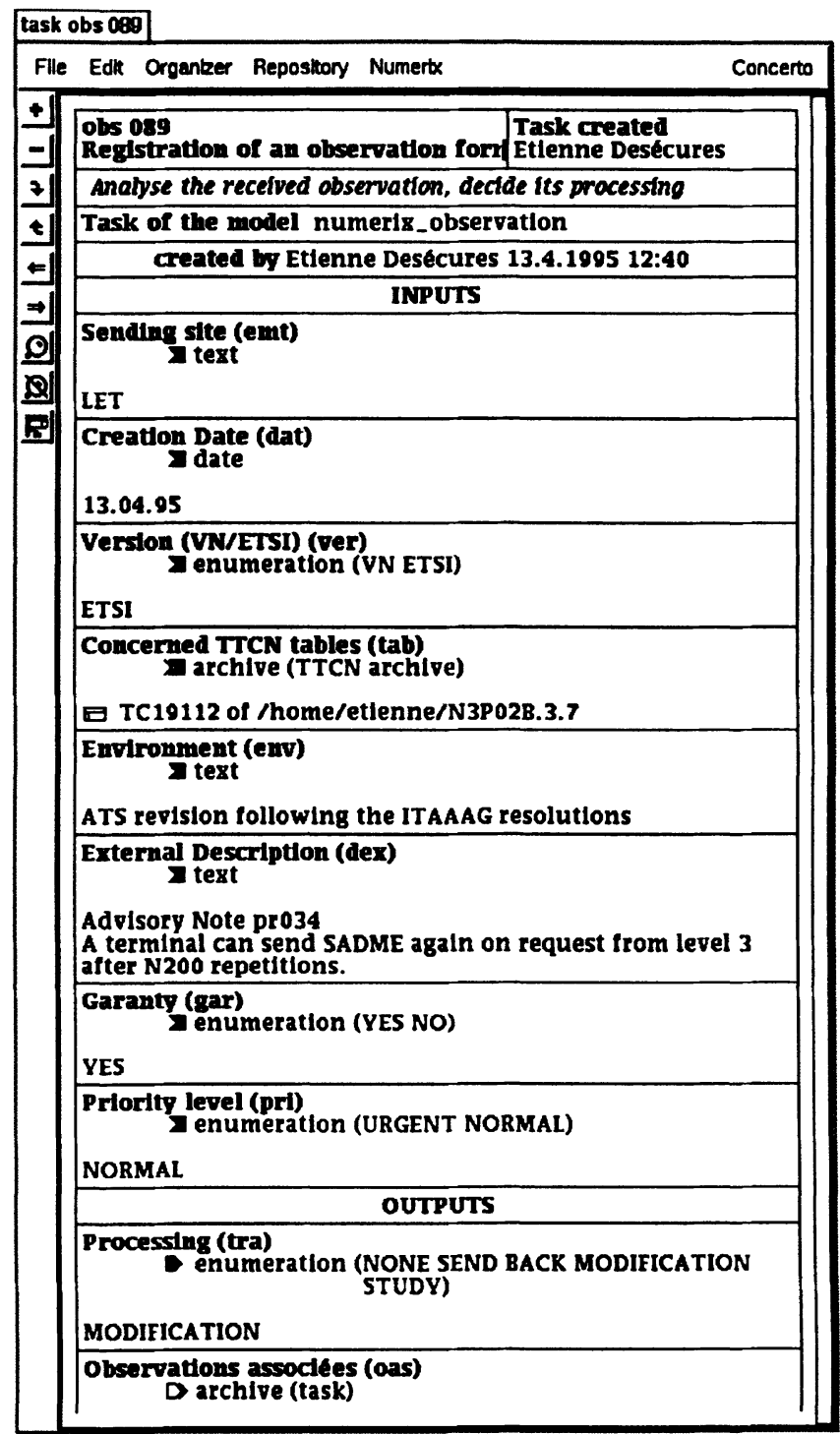

Figure 2 Example of an Observation task description form

\subsection{Modification tasks}

Every modification undertaken on an ATS is declared in a modification task. The various fields contain: 
- hypertext links to the tables to be corrected and also to the tables effectively corrected,

- hypertext links to the observation task which initiates the processing, and its associated observation tasks,

- a complete description of the modifications carried out.

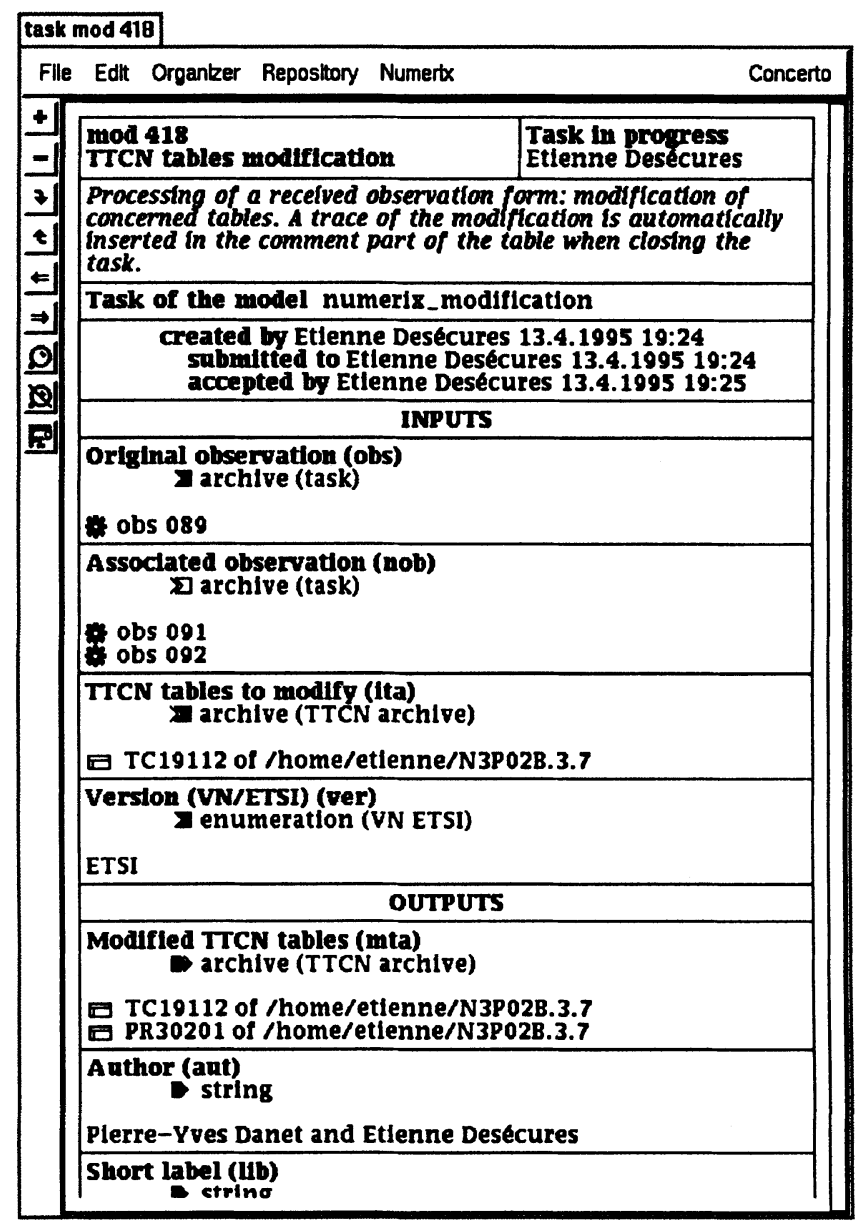

Figure 3 Example of a Modification task description form

The "create modification" command is used to create a modification task from an observation task. The elements of the observation task are automatically reported in the input fields of the modification task.

The Numerix tool can be used to create a document from a modification task. 


\subsection{History lists}

The complete history list of a given table is automatically built by Numerix: every completion of a modification task inserts automatically a history statement in the Detailed Comments field of the modified TTCN table taken from the modification task.

\begin{tabular}{|c|c|c|c|c|c|}
\hline \multicolumn{6}{|c|}{ Test Case Dynamic Behaviour } \\
\hline \multicolumn{2}{|c|}{$\begin{array}{l}\text { Test Case Name: } \\
\text { Group: } \\
\text { Purpose: } \\
\text { Configuration: } \\
\text { Default: } \\
\text { Comments: }\end{array}$} & \multicolumn{4}{|c|}{$\begin{array}{l}\text { TC19114 } \\
\text { ISDN/Basic_call/Successful/Speech/ } \\
\text { GLOBAL CALL RER. - STATE R1 - PASSIVE IUT BEHAVIOUR } \\
\text { SYNTACTICALLY INVALID TEST EVENTS } \\
\text { DF69902 }\end{array}$} \\
\hline $\mathbf{N r}$ & Label & Behoviour Description & Constraints Ref & Verdict & Comments \\
\hline $\begin{array}{l}4 \\
5 \\
6\end{array}$ & L1 & $\begin{array}{l}\text { + PR39003 } \\
\text { LIERROR START TAC } \\
\text { L2STATUSr [(STATUS.CST.CSTV = 61) } \\
\text { AND (STATUS.CAU.CVAL = 96)] CAN- } \\
\text { CEL TAC } \\
\text { + CS59003 }(61,1) \\
\text { + UMS9902 } \\
\text { GOTO L1 }\end{array}$ & $\begin{array}{l}\text { ERR225 } \\
\text { ST7 (0) }\end{array}$ & (P) & $\begin{array}{l}\text { preamble to RO } \\
\text { PDU RESTART ACK } \\
\text { valid STATUS } \\
\text { unexpected message }\end{array}$ \\
\hline \multicolumn{6}{|c|}{ 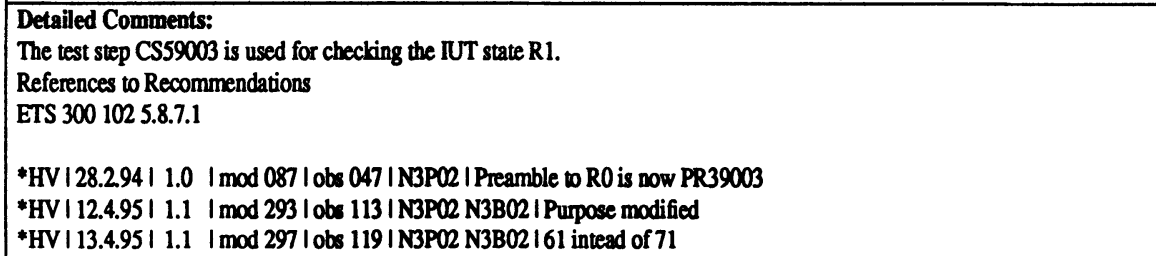 } \\
\hline
\end{tabular}

Figure 4 Example of a TTCN table with a history list which automatically results from modification form processing

Each statement is composed of the modification task main elements: date, version, observation and modification task numbers, embedding suites, short description of the modification. The history list which appears in each modified table can be immediately consulted in every representation of the table (MP, screen, paper). Obviously, these statements do not garble actual comments, which remain modifiable.

The Numerix tool can be used to create a document containing the history list in a more readable way from a given TTCN table.

\subsection{Delivery processing}

With a single command the Numerix tool allows the production of:

- a delivery document

- a Unix file containing the suite MP. 
A modification delivery document contains the set of modification forms that initiated the delivery. A full ATS delivery document contains the GR format of the whole ATS delivered, followed by the list of modification forms. In both cases, modifications are described starting at a chosen version of the ATS ; it may be the last one delivered or a previous one, so that specific deliveries can be done for particular user sites.

Generally the delivery of a suite makes official a new version of that suite. It is important to freeze this new version. Then it is exported to a specific Concerto base and can be consulted through the network by the using sites.

\subsection{Version management and ATS composition}

The TTCN database is split in two separate areas:

- the "Reference Area",

- the "Development Area".

The reference area contains the stable versions of the ATSs while the ATSs under development or modification are stored in the development area. ATSs in the reference area are frozen: they are write-protected. A modification to a frozen suite implies first the creation of an unfrost copy placed in the development area with a new version number. This operation is called "check-out". The "check-in" operation consists in moving the modified ATS from the development area to the reference area and to freeze it. The link between an ATS version and the previous version is kept. This mechanism, supported by the Configurator environment, is widespread in Concerto and is also applicable to shared fragments.

ATSs can be composed of fragments shared by other suites. These fragments are called ATS components. The current practice at CNET is to use components at the table level, but it could be done at any other level, e.g. Test Groups. Modifications to tables usually lead to the creation of one or several versions of the tables. Before delivering an ATS it is necessary to update it, so that it refers the appropriate, usually the newer, version of every component. This operation is assisted by a special command allowing to choose in a matrix which version of each component is to be taken. The updated ATS can then be frozen and constitutes a stable version which can be delivered and exported to other sites.

\section{CONCLUSION}

The Numerix solution installation is in progress at CNET. It is too early to make definite conclusions but it can be said that:

- The saving of all the significant events in the organizer database and the facilities to query this database is appreciated by the users,

- The sharing of tables between ATSs allows the reduction of the number of managed tables from 60000 to 20000 .

- The use of simple and familiar concepts (observation, modification, etc.) enables the users to be operational very quickly,

- Numerix has been designed to be, as far as possible, not too much linked to the method and parameterizable. It could be easily adapted to support another method. 


\section{REFERENCES}

ISO/9646-1 : 1991, Information technology - Open Systems Interconnection - Conformance testing methodology and framework - Part 1: General concepts

ISO/9646-2 : 1991, Information technology - Open Systems Interconnection - Conformance testing methodology and framework - Part 2: Abstract test suite specification

Pierre-Yves Danet : Management and maintenance of TTCN Abstract Test Suites ; OSTC - 9 March 1995

Concerto/Numerix User Manual v1.0 